LBNL-54529

\title{
Massless Flavor in Geometry and Matrix Models
}

\author{
Radu Roiban ${ }^{\mathrm{a}}$, Radu Tatar ${ }^{\mathrm{b}}$ and Johannes Walcher ${ }^{\mathrm{c}}$ \\ ${ }^{a}$ Department of Physics \\ University of California, Santa Barbara, CA 93106 \\ ${ }^{\mathrm{b}}$ Department of Physics, 366 Le Conte Hall \\ University of California, Berkeley, CA 94720 \\ and \\ Lawrence Berkeley National Laboratory \\ Berkeley, CA 94720 \\ ${ }^{c}$ Kavli Institute for Theoretical Physics \\ University of California, Santa Barbara, CA 93106
}

This work was supported in part by the Director, Office of Science, Office of High Energy Physics, of the U.S. Department of Energy under Contract No. DE-AC03-76SF00098.

\section{DISCLAIMER}

This document was prepared as an account of work sponsored by the United States Government. While this document is believed to contain correct information, neither the United States Government nor any agency thereof, nor The Regents of the University of California, nor any of their employees, makes any warranty, express or implied, or assumes any legal responsibility for the accuracy, completeness, or usefulness of any information, apparatus, product, or process disclosed, or represents that its use would not infringe privately owned rights. Reference herein to any specific commercial product, process, or service by its trade name, trademark, manufacturer, or otherwise, does not necessarily constitute or imply its endorsement, recommendation, or favoring by the United States Government or any agency thereof, or The Regents of the University of California. The views and opinions of authors expressed herein do not necessarily state or reflect those of the United States Government or any agency thereof or The Regents of the University of California. 
UCB-PTH-03/03

NSF-KITP-03-10

hep-th/0301217

\title{
Massless Flavor in Geometry and Matrix Models
}

\author{
Radu Roiban ${ }^{a}$, Radu Tatar ${ }^{b}$ and Johannes Walcher ${ }^{c}$ \\ ${ }^{a}$ Department of Physics \\ University of California, Santa Barbara, CA 93106 \\ ${ }^{b}$ Department of Physics, 366 Le Conte Hall \\ University of California, Berkeley, CA 94720 \\ and \\ Lawrence Berkeley National Laboratory \\ Berkeley, CA 94720 \\ ${ }^{c}$ Kavli Institute for Theoretical Physics \\ University of California, Santa Barbara, CA 93106
}

\begin{abstract}
The proper inclusion of flavor in the Dijkgraaf-Vafa proposal for the solution of $\mathcal{N}=1$ gauge theories through matrix models has been subject of debate in the recent literature. We here reexamine this issue by geometrically engineering fundamental matter with type IIB branes wrapped on non-compact cycles in the resolved geometry, and following them through the geometric transition. Our approach treats massive and massless flavor fields on equal footing, including the mesons. We also study the geometric transitions and superpotentials for finite mass of the adjoint field. All superpotentials we compute reproduce the field theory results. Crucial insights come from T-dual brane constructions in type IIA.
\end{abstract}

January 2003

\footnotetext{
E-mail: ${ }^{a}$ radu@vulcan2 .physics .ucsb.edu

${ }^{b}$ rtatar@socrates. berkeley.edu

$c_{\text {walcher@kitp.ucsb.edu }}$
} 


\section{Contents}

1 Introduction 1

1.1 Field theorv results for arbitrary mass for the adjoint field . . 4

2 Engineering of massive adioint fields and massless flavors 5

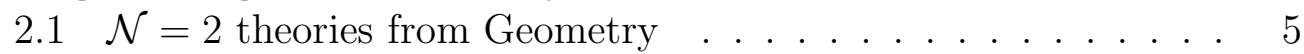

$2.2 \mathcal{N}=1$ theories from geometrv: massless quarks $\ldots \ldots \ldots 7$

3 Geometric transition with fundamental fields 13

4 Effective superpotential at strong coupling 16

5 Effective superpotential at weak coupling: Matrix Models 23

5.1 Review of the results for pure gauge theories . . . . . . . . . 23

5.2 Massive and massless matrix models . . . . . . . . . . . . . 24

6 Conclusions 28

\section{Introduction}

In the last years many steps have been taken toward a better understanding of the dualities between field theory and string theory. One direction was initiated in [1] and consisted in studying the large $\mathrm{N}$ dualities in the context of type A topological strings. This topological duality was embedded in the physical superstring theory in [2] and then further developed in [3, 4, 5, [6, 7, 8, 9] (see also [10, 11, 12] for an alternative approach, involving brane configurations). The main result of these studies was a method of calculating the effective superpotential of a four-dimensional field theory using aspects of the flux configurations and of the geometry of the compact/transverse space.

It is natural to suspect that similar dualities exist for the type B topological strings on Calabi-Yau manifolds. They have been discussed in a series of papers by Dijkgraaf and Vafa 14, 15. On the closed string side of the duality the effective superpotential of the four-dimensional gauge theory is generated by the Gukov-Vafa-Witten (GVW) superpotential [3]. On the open string side of the duality the four-dimensional gauge theory is realized by wrapping D-branes on certain 2-cycles and the effective superpotential is generated by the topological open string theory living on these cycles. This is described by 
the holomorphic Chern-Simons theory which becomes a simple matrix model with the potential given by the superpotential of the gauge theory. Building on these results, a stronger claim has been argued in [16], stating that for a class of $\mathcal{N}=1$ theories, with fields in the adjoint and bifundamental representations of the gauge group, the effective superpotential can be expressed in terms of the planar free energy of this matrix model. Using field theory techniques it was shown that, for models with one chiral field $\Phi$ in the adjoint representation and a tree level superpotential $W(\Phi)$, the truncation to planar diagrams appeared due to the holomorphy of the expected effective action [18, and/or to the cancellation of dependence on momenta between the bosonic and fermionic integrals [17.

It is interesting to extend the original arguments of Dijkgraaf and Vafa to include fields in other representations of the gauge group. The case of fields in the fundamental representation was discussed extensively in [20]-39] (see also [40] for progress in other directions), in general without reference to any possible string theory realization of such theories ${ }^{1}$. It turns out that the matrix/gauge theory relation implies that the flavor contribution to the effective superpotential is exactly taken into account by the one-boundary free energy of the corresponding matrix model [21, 25, 30, 37.

It is however rather difficult to extend these results to theories with massless flavor fields. Indeed, in this case the low energy theory is not described only in terms of the glueball superfield as the naive matrix model predicts, but it must also contain quark bilinears. An attempt to handle this case was introduced in [24] and requires the introduction of delta functions relating the matrix model flavor fields with the corresponding gauge theory mesons. An alternate suggestion was presented in 30] and involves deforming the naive matrix model by mass terms for all massless fields and then computing the superpotential from the one-boundary free energy. The gauge theory superpotential is obtained by integrating in, in the gauge theory sense, the fields that were originally massless and then taking the massless limit. Finally, these two procedures were shown to be equivalent in 39. There the gauge theory meson field is identified with the Lagrange multiplier enforcing the massless limit.

In this paper we reconsider the geometric arguments which led to the matrix model/gauge theory duality. First we generalize the results of [3] to

\footnotetext{
${ }^{1}$ Exceptions are references [31] and 35, where the approach considered is different from the one we use in our paper
} 
the case of an adjoint field of arbitrary mass as well as to the case of massless quarks. As the results of 14, 15, 16] were based on geometric transitions relating open and closed string theories, our new results shed a new light on the matrix/gauge theory relation in the presence of massless fields. We use the T-dual brane configurations [10, 11, 12, 13, to understand the dynamics of the field theory, as the mass of the adjoint field as well as the mass and vevs of the fields transforming in the fundamental representation of the gauge group can be read from the positions of the different branes.

It is important to stress that in our treatment the flavor fields are described by D5 branes wrapping noncompact two-cycles and these branes exist on both sides of the transition. This is very similar to the situations encountered in the analysis of defect CFT-s [53]. On the open string theory side the gauge theory is realized on the common part of the world volume of D5 branes wrapping the compact $\mathbb{P}^{1}$ cycle of the small resolution of the conifold and of D5 branes wrapping the noncompact $\mathbb{P}^{1}$ cycles. Because of the noncompactness of the D5 branes wrapping the noncompact cycles the open strings stretching between them yield no dynamical fields. On this side of the duality the gauge theory effective superpotential will be generated by the dynamics of open strings governed by the holomorphic Chern-Simons theory 43].

After the geometric transition (which corresponds to the strong coupling limit of gauge theory) the D5 branes wrapping the compact cycles are replaced by flux. The branes wrapping noncompact cycles survive the transition and can be interpreted as probes of the deformed geometry with flux. They give rise to dynamical fields which, roughly speaking (we will make this precise later), can be identified with the gauge theory mesons. In this formulation of the gauge theory the effective superpotential receives two conceptually different contributions. The first part is the flux-generated superpotential while the second part is given by the dynamics of open strings starting and ending on the remaining D5 branes. This latter part reproduces the results of 21] for massive flavor fields as well as the ones previously obtained in 46] for the massless ones.

Finally, we explain the appearance of the delta function identifying the gauge theory meson and the matrix model quark bilinear both from a topological string perspective as well as using the M5 brane dynamics.

Before proceeding in the following sections with the geometric analysis let us first summarize the gauge theory results we will recover, namely the superpotential for an $\mathcal{N}=1$ gauge theory with both massive and massless 
quarks, a massive field in the adjoint representation of the gauge group and Yukawa interactions.

\subsection{Field theory results for arbitrary mass for the adjoint field}

Consider an $\mathcal{N}=2$ theory with gauge group $U\left(N_{c}\right)$ and massive and massless quarks and consider breaking supersymmetry to $\mathcal{N}=1$ by turning on arbitrary mass term for the adjoint chiral multiplet as well as allowing the Yukawa coupling to be different from the gauge coupling. Denoting the massive quarks by $Q^{(1)}$ and $\tilde{Q}^{(1)}$, the tree level superpotential is:

$$
W=\sqrt{2} g \operatorname{Tr}[\tilde{Q} \phi Q]+m \operatorname{Tr}\left[Q^{(1)} \tilde{Q}^{(1)}\right]+\mu \operatorname{Tr} \phi^{2}
$$

To discuss the Higgs branch of this theory one first integrates out the adjoint field $\phi$. The renormalization group fixes the dynamical scale of the resulting theory to be

$$
\Lambda_{\mathcal{N}=1}^{3 N_{c}-N_{f}}=\mu^{N_{c}} \Lambda_{\mathcal{N}=2}^{2 N_{c}-N_{f}}
$$

while standard nonrenormalization theorem arguments imply that perturbatively the superpotential is just

$$
W=\frac{g^{2}}{2 \mu} \operatorname{Tr}[(Q \tilde{Q})(Q \tilde{Q})]+m \operatorname{Tr}\left[Q^{(1)} \tilde{Q}^{(1)}\right]
$$

For $\mu \rightarrow \infty$, this superpotential approaches zero and we then obtain $\mathcal{N}=1$ SQCD.

Using symmetry and holomorphy arguments as well as smoothness in the limit $g \rightarrow 0$ one can show that, if $N_{f} \leq N_{c}-1$, the effective superpotential of this theory is just

$$
W=\frac{g^{2}}{2 \mu} \operatorname{Tr}[(Q \tilde{Q})(Q \tilde{Q})]+m \operatorname{Tr}\left[Q^{(1)} \tilde{Q}^{(1)}\right]+\left(N_{c}-N_{f}\right)\left[\frac{\Lambda_{\mathcal{N}=1}^{3 N_{c}-N_{f}}}{\operatorname{det}(Q \tilde{Q})}\right]^{\frac{1}{N_{c}-N_{f}}}
$$

where the last term represents the nonperturbative contributions. At finite values for the mass of the adjoint field and generic values of the mass of the quarks, the expectation value of the meson field $M_{i j}=Q_{i} \tilde{Q}_{j}$ has two types of diagonal entries [46. As the mass of the adjoint field is taken to infinity all these vacua run away to infinity and there is no vacuum left at finite distance in the moduli space of $\mathcal{N}=1$ SQCD with massless quarks. 
As stated previously, we will recover the superpotential (41) (and thus all its consequences) from geometric considerations. We also find a geometric interpretation of the "integrating in/out method" of [42. To achieve this, we will begin by discussing the small resolution of the conifold in the case of finite adjoint mass.

\section{Engineering of massive adjoint fields and massless flavors}

In this section we describe the details of the geometric engineering of field theories with an adjoint field of finite mass and massive and massless flavor fields. We begin by reviewing some results of [12] concerning the construction of $\mathcal{N}=2$ field theories as well as the addition of fields in the fundamental representation of the gauge group. We then proceed to break supersymmetry by a finite mass for the adjoint field as well as to find the geometric interpretation of the gauge theory meson field. In the next section we will discuss the geometric transition of this setup.

\section{1 $\mathcal{N}=2$ theories from Geometry}

The field theory of interest is realized on the world volume of (fractional) branes whose transverse space is the tensor product of an ADE singularity with a two-dimensional plane. The resolved space contains a collection of $\mathbb{P}^{1}$ cycles, together with their normal bundles. For each cycle this is $\mathcal{O}(-2) \oplus$ $\mathcal{O}(0)$. The $\mathcal{O}(0)$ fibers represent the Coulomb branch of the gauge theory. Inclusion of fields in the fundamental representation of the gauge group as well as breaking of supersymmetry to $\mathcal{N}=1$ by a finite mass parameter for the chiral multiplet in the $\mathcal{N}=2$ vector multiplet is, to some extent, clearer in the brane realization of the theory. We will summarize this description which is related to the geometric one by $T$-duality. For this we need to examine in slightly more detail the geometric description.

The total space of the normal bundle over the $i$-th $\mathbb{P}^{1}$ can be covered with two patches $\mathbb{C}_{i, S}^{3}$ and $\mathbb{C}_{i, N}^{3}$, where $N$ and $S$ refer to the North and South pole of the corresponding $\mathbb{P}^{1}$ cycle. The transition functions are given by

$$
Z_{i}^{\prime}=\frac{1}{Z_{i}} \quad Y_{i}^{\prime}=Y_{i} Z_{i}^{2} \quad X_{i}^{\prime}=X_{i}
$$


Clearly, the coordinate $X_{i}$ parameterizes the trivial fibers $\mathcal{O}(0)$ while the remaining coordinates describe the total space of $\left.\mathcal{O}(-2)\right|_{\mathbb{P}^{1}}$. To plumb the set of $\mathbb{P}^{1}$ cycles (together with their normal bundles) and reconstruct the full space one uses certain identifications dictated by the ADE Dynkin diagram associated to the chosen singularity.

An $\mathcal{N}=2$ field theory is constructed by wrapping D5 branes on the $\mathbb{P}^{1}$ cycles. The precise interactions of this theory depend on the singularity we started with, i.e. they depend on the intersection of the $\mathbb{P}^{1}$ cycles. For example, in the case of a resolved $A_{n}$ singularity, the $n$ copies of $\left.\mathcal{O}(-2)\right|_{\mathbb{P}^{1}}$ are connected by the identification

$$
Y_{i}^{\prime} \rightarrow Z_{i+1} \quad Z_{i}^{\prime} \rightarrow Y_{i+1} \quad X_{i}^{\prime} \rightarrow X_{i+1}
$$

which means that the north pole of the i-th $\mathbb{P}^{1}$ cycle meets the south pole of the i+1-th $\mathbb{P}^{1}$ cycle. By considering such a singularity together with $N_{i}$ D5 branes on the i-th $\mathbb{P}^{1}$ cycle we have a gauge theory with gauge group $\prod_{i=1}^{n} U\left(N_{i}\right)$ and hypermultiplets $F_{i}, \tilde{F}_{i}, i=1, \cdots, n-1$ in the bifundamental representations $\left(N_{i}, \bar{N}_{i+1}\right)$ and $\left(\bar{N}_{i}, N_{i+1}\right)$ respectively, as well as a superpotential consisting of Yukawa interactions of the fields in $\left(N_{i}, \bar{N}_{i+1}\right),\left(\bar{N}_{i}, N_{i+1}\right)$ and $\left(N_{i+1}, \bar{N}_{i+1}\right)$.

To translate the geometrical picture into a brane configuration we split the angular and radial directions of the $\mathbb{P}^{1}$ cycles, and we reduce the geometrical picture to one where the angular direction is removed. This means considering a "skeleton" of the geometrical picture. This can be achieved by a T-duality [10, 11, 12. The T-duality direction is a circle action on the normal bundle over the $\mathbb{P}^{1}$ cycle, given by

$$
\begin{array}{r}
Z_{i} \rightarrow e^{i \theta} Z_{i}, \quad Y_{i} \rightarrow e^{-i \theta} Y_{i} \\
Z_{i}^{\prime} \rightarrow e^{-i \theta} Z_{i}^{\prime}, \quad Y_{i}^{\prime} \rightarrow e^{i \theta} Y_{i}^{\prime},
\end{array}
$$

whose orbits degenerate ${ }^{2}$ along $Z_{i}=Y_{i}=0$ and $Z_{i}^{\prime}=Y_{i}^{\prime}=0$. By using [50, the lines of singularity get mapped into $n$ parallel NS5 branes. The $N_{i}$ D5 branes wrapped on the blown-up $\mathbb{P}_{i}^{1}$ cycle are mapped into $N_{i}$ D4 branes suspended between the $i$-the and $(i+1)$-th NS5 brane.

In the present discussion we are interested in the inclusion of fields transforming in the fundamental representation of the gauge group. In the brane realization of the theory such fields are introduced by including semi-infinite

\footnotetext{
${ }^{2}$ By abuse of terminology we will call these degenerate orbits "lines of singularity".
} 
D4 branes which end on the NS branes. Let us consider that we have $N_{f, i}$ flavors in the fundamental representation of $U\left(N_{i}\right)$ and denote these fields by $Q_{i}, \tilde{Q}_{i}, i=1, \cdots, n$. If the fundamental flavors are massive, we denote their masses by $m_{i}$. They are given by the distance along the NS5 branes between the endpoint of the corresponding semi-infinite D4 brane and the D4 branes describing the $U\left(N_{i}\right)$ part of the gauge group.

With this starting point it is easy to construct the geometric version of the setup by performing the inverse of (17). The result is that in the geometric picture the fields transforming in the fundamental representation of the gauge group are introduced as D5 branes wrapping non-compact holomorphic 2cycles given by:

$$
Y_{i}=0 \quad \text { or } \quad Y_{i}^{\prime}=0, \quad X=m_{i}
$$

The choices $Y_{i}=0$ or $Y_{i}^{\prime}=0$ are identical, as they describe the same point in the total space.

\section{$2.2 \mathcal{N}=1$ theories from geometry; massless quarks}

We now deform the geometry by adding superpotentials for the adjoint fields (including mass terms). Generic theories without matter fields have been analyzed in detail in [12. We discuss here the simplest model, obtained by adding just the mass term for the adjoint chiral multiplet in the $\mathcal{N}=2$ vector multiplets, which breaks supersymmetry to $\mathcal{N}=1 .^{3}$ The superpotential will therefore be:

$$
\begin{aligned}
W & =\sum_{i=1}^{n}\left(\frac{m}{2} \operatorname{Tr} \Phi_{i}^{2}\right. \\
& \left.+\operatorname{Tr}\left(F_{i} \Phi_{i+1} \tilde{F}_{i}-\tilde{F}_{i+1} \Phi_{i} F_{i+1}+\lambda_{i} Q_{i} \Phi_{i} \tilde{Q}_{i}+\lambda_{i}^{\prime} Q_{i+1} \Phi_{i+1} \tilde{Q}_{i+1}\right)\right)
\end{aligned}
$$

where $\lambda_{i}$ and $\lambda_{i}^{\prime}$ are $\sqrt{2} g$ if the Yukawa interactions are to preserve $\mathcal{N}=2$ supersymmetry, but can have arbitrary values for the $\mathcal{N}=1$ theories.

For a better understanding we begin by considering a theory with gauge group $U(N)$ and $N_{f}$ fields in the fundamental representation, with mass parameters $m_{i}$. Before supersymmetry breaking, this is the world volume

\footnotetext{
${ }^{3}$ In this section we consider the field theory and geometry deformations when the mass for the adjoint chiral multiplet is finite or infinite. A similar discussion appeared in 54 for the case of branes probing singularities, whereas in our case we deal with D5 branes wrapped on blown-up cycles.
} 
theory of $N$ D5 branes wrapped on the nontrivial $\mathbb{P}^{1}$ cycle of a blown up $A_{1}$ singularity and $N_{f}$ D5 branes wrapped on the noncompact cycles defined by

$$
Y=0, X=m_{i} \quad i=1, \ldots, m
$$

or

$$
Y^{\prime}=0, X=m_{i} \quad i=1, \ldots, p
$$

with

$$
m+p=N_{f} .
$$

The brane configuration corresponding to this geometry is constructed out of two parallel $N S 5$ branes with $N$ D 4 branes suspended between them as well as $m$ and $p$ semi-infinite D4 branes ending on the left and right $N S 5$ brane, respectively. In this language supersymmetry breaking is realizes by rotating the NS5 branes relative to each-other. The rotation angle is a function of the mass of the adjoint field.

The T-duality described in the previous section provides the connection between the rotated brane configuration and geometry. Roughly speaking, rotating the $N S 5$ branes corresponds to fibering the $A_{1}$ singularity over the dimensional plane. In other words, the normal bundle of the blown up $\mathbb{P}^{1}$ is modified. The fields transforming in the fundamental representation are still described by D5 branes wrapping noncompact cycles. Unlike the situation above, after the rotation the two choices of cycles become physically inequivalent.

Two limits of geometry as a function of the mass of the adjoint are important to discuss:

- $\mathbb{P}^{1}$ with normal bundle $\mathcal{O}(-2) \oplus \mathcal{O}(0)$, obtained for a zero mass for the adjoint field. In this limit the field theory has $\mathcal{N}=2$ supersymmetry, as discussed in the previous section.

- $\mathbb{P}^{1}$ with normal bundle $\mathcal{O}(-1) \oplus \mathcal{O}(-1)$ (the resolved conifold), obtained for an infinite mass for the adjoint field. In this limit the field theory in the world volume of the D5 branes is $\mathcal{N}=1$ SQCD limit, i.e. the field in the adjoint representation is decoupled.

This latter choice is described by two copies of $\mathbb{C}^{3}$, parametrized by $(X, Y, Z)$ and $\left(X^{\prime}, Y^{\prime}, Z^{\prime}\right)$, together with the transition function:

$$
Z^{\prime}=Z^{-1}, X^{\prime}=X Z, Y^{\prime}=Y Z
$$


As discussed in [10], the singular conifold is recovered through the blowdown map

$$
x=X=X^{\prime} Z^{\prime}, y=Z Y=Y^{\prime}, u=Z X=X^{\prime}, v=Y=Z^{\prime} X^{\prime}
$$

which implies that

$$
x y-u v=0
$$

which defines the conifold at the singular point. This map together with (77) induce a circle action on the coordinates in the two patches which can be used to translate between the brane and geometric description:

$$
\begin{aligned}
& Z \rightarrow e^{i \theta} Z, \quad X \rightarrow X, \quad Y \rightarrow e^{-i \theta} Y \\
& Z^{\prime} \rightarrow e^{-i \theta} Z^{\prime}, X^{\prime} \rightarrow e^{i \theta} X^{\prime}, \quad Y^{\prime} \rightarrow Y^{\prime} .
\end{aligned}
$$

The lines of singularity are $Z=Y=0$ in the first $\mathbb{C}^{3}$ and $Z^{\prime}=X^{\prime}=0$ in the second $\mathbb{C}^{3}$, which are clearly orthogonal. Thus, the brane configuration corresponding to the small resolution of the conifold contains two orthogonal NS5 branes.

Let us now analyze the fields in the fundamental representation. As we discussed before, they correspond to D5 branes wrapping non-compact holomorphic cycles

$$
Y=0, \quad X=m
$$

or to D5 branes wrapping a non-compact holomorphic 2-cycles

$$
X^{\prime}=0, \quad Y^{\prime}=m
$$

Thus, we notice that after the $A_{1}$ singularity was fibered over the transverse two dimensional space, these two cycles are no longer equivalent. Indeed, as the lines of singularity in the geometry are now along the $X$ and $Y^{\prime}$ directions, after a T-duality on the above orbit we get two orthogonal NS branes on the directions $X$ and $Y^{\prime}$. The D5 branes wrapped on the compact $\mathbb{P}^{1}$ are mapped into finite D4 branes (between the two orthogonal NS branes) while the ones on the non-compact holomorphic cycles map into semi-infinite D4 branes which can end on one NS brane or the other.

There are also $\mathcal{N}=1$ brane configurations (and geometries) which correspond to finite masses for the adjoint field. As we stated above, in terms of brane configurations this means that the NS branes are neither parallel nor 
orthogonal. By a T-duality we can add a circle to this "geometric skeleton" and obtain a geometry where the lines of singularity are neither parallel nor orthogonal. To make this more concrete, the transition function $X^{\prime}=X Z$ is replaced with $X^{\prime}=X_{r} Z$ where $X_{r}$ is some function of $X, Y$ and $Z$. Thus, the geometry is now:

$$
Z^{\prime}=\frac{1}{Z}, \quad X^{\prime}=X_{r} Z, \quad Y^{\prime}=Y Z
$$

Taking

$$
X_{r}=X-\frac{1}{m_{a d j}} Y Z
$$

and using the blowdown map (14) we find the following deformation of the singular conifold:

$$
u v-y\left(x-\frac{1}{m_{a d j}} y\right)=0 .
$$

In the limit of infinite $m_{a d j}$ we recover the usual conifold geometry while in the limit of vanishing $m_{\text {adj }}$ rescaling $u$ and $v$ leads to the $A_{1} \times \mathbb{C}$.

The orbit (16) has the form:

$$
\begin{array}{r}
Z \rightarrow e^{i \theta} Z, \quad X_{r} \rightarrow X_{r}, \quad Y \rightarrow e^{-i \theta} Y \\
Z^{\prime} \rightarrow e^{-i \theta} Z^{\prime}, \quad X^{\prime} \rightarrow e^{i \theta} X^{\prime}, \quad Y^{\prime} \rightarrow Y^{\prime},
\end{array}
$$

and we observe that the degeneration is indeed along the union of complex lines along $X_{r}$ in the first $\mathbb{C}^{3}$ and $Y^{\prime}$ in the second $\mathbb{C}^{3}$. As promised, Tduality on this orbit produces a configuration of two NS5 branes at an angle determined by $X_{r}$.

We can reach a similar result by starting with the $\mathcal{N}=2$ geometry (5) (with $i=1$ ) and deforming the transition functions to

$$
Z^{\prime}=1 / Z, \quad Y^{\prime}=Y Z^{2}+m_{A} X Z .
$$

To see what happens when we vary the mass of the adjoint field, we switch again to the map (14) and find

$$
u v-y^{2}+m_{A} x y=0,
$$

which is the same equation we had before, up to a rescaling of $u$ and $v$.

The geometric transition takes us to a deformed conifold. Since there exists a holomorphic change of coordinates which casts equation (24) into 
that of the conifold, one may say that the two geometries describe the same physics. This is, however, not the case as various boundary conditions change under these transformations. Anticipating later arguments, the boundary conditions can be naturally chosen in one coordinate system while the computations are easier in the other one; the coordinate transformation will introduce a dependence on the mass of the adjoint field in the boundary conditions.

Up to now we have discussed the geometric construction of massive fields in the fundamental representation of the gauge group. Our main goal is, however, to find a geometric description of massless matter fields. To reach this goal we start with the brane configuration describing such fields 44 and then subject it to T-duality transformations along the orbit (22).

There are two choices of introducing matter, one with semi-infinite D4 branes and the other with D6 branes. In the following we will use D4 branes for this purpose and begin by describing the setup at vanishing string coupling, when all branes are represented by straight hyperplanes.

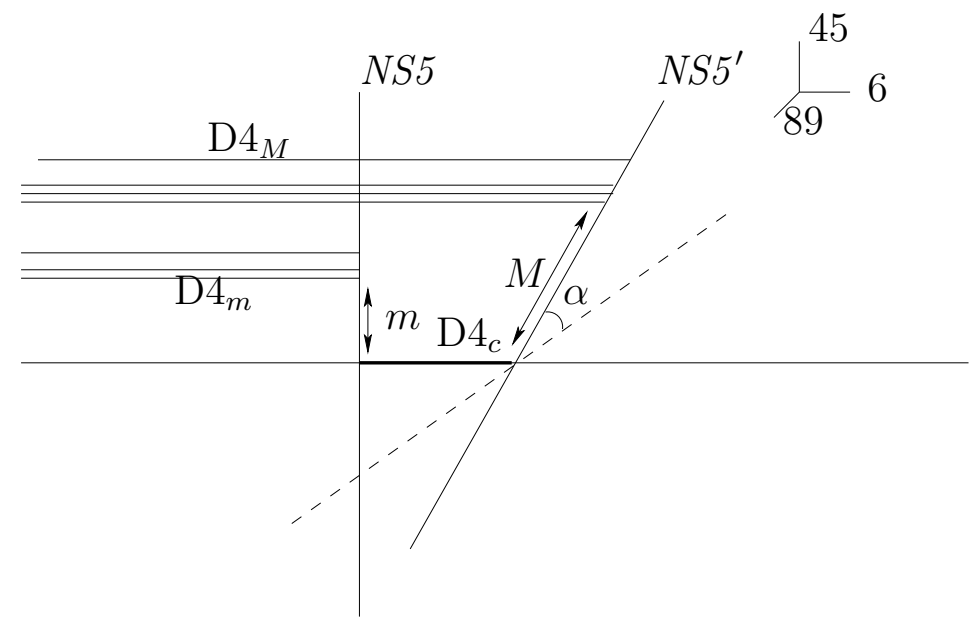

Figure 1: Brane construction .

To be specific, we consider the configuration in figure 1. The dashed line represents the directions orthogonal on the (456) space. The fact that the $N S^{\prime}$ brane is not orthogonal on this 3-space corresponds in field theory language to introducing a superpotential quadratic in the chiral superfield transforming in the adjoint representation of the gauge group. This can be 
easily seen using symmetry arguments [46, 47]. The $N=2$ theory is invariant under $U(1) \times S U(2) R$-symmetry which correspond to rotations in the (45) and (789) directions, respectively. In the rotated brane configuration $S U(2)$ is broken to $U(1)$ corresponding to rotations along the $N S^{\prime}$ directions. Denoting by $x$ the coordinate along the $N S$ brane and by $y$ the coordinate along the $N S^{\prime}$ one, it follows that they are proportional and that the proportionality coefficient is charged under both $U(1) R$-symmetry groups, with charges of the same magnitude and opposite sign. The only field theory object with these properties is the mass of the adjoint field.

Let us now discuss the interpretation of the position of the end of the D4 branes on the $N S$ and $N S^{\prime}$ branes. Separating the D4 branes induces a breaking of the $U\left(N_{f}\right)$ flavor symmetry. If the separation is in the $(4,5)$ direction, then it should be interpreted as a field theoretic mass term for the quarks as this is the only parameter charged under the corresponding $U(1)$ symmetry. If the separation is along the $N S^{\prime}$ brane this should be interpreted as breaking due to a nonvanishing eigenvalue for the meson field. Indeed, this is the only field theoretic object charged under the second $U(1)_{R}$ symmetry.

At vanishing string coupling, it is possible to understand from figure 1 the phenomena which occur when one changes the description of the theory from having a massive field to having a bilinear in that field with non vanishing expectation value. Indeed, all one has to do is to transform a $\mathrm{D} 4_{m}$ brane into a $\mathrm{D} 4_{M}$ one. This is possible only by recombining the $\mathrm{D} 4_{m}$ brane with one of the color branes. Under this operation the gauge group is spontaneously broken, as at vanishing (string) coupling the only way for a bilinear in fields to have vev is for each of the two factors to have a vev. Thus, in the process of changing the description of the theory from having a massive field to having a bilinear with non vanishing vev, the rank of the unbroken gauge group decreases with the number of $\mathrm{D} 4_{m}$ branes transformed into $\mathrm{D} 4_{M}$. This recombination of different types of $\mathrm{D} 4$ branes can be interpreted as the analog of the field-theoretic "integrating in/out" procedure of 42 .

At finite coupling it is certainly possible for a composite operator to acquire a non vanishing vev without its building blocks having one. However, if the vev is larger than the dynamical scale of the theory, the vev can be treated classically and thus, in the case of quark bilinears, leads to a spontaneous breaking of the gauge group as well. We will return to this in a later section and quantitatively recover this picture from a geometric description.

This configuration can be easily mapped to the type IIB configuration. As discussed earlier, the $N S$ branes and the compact D4 branes are mapped 
to the resolved conifold in coordinates (21); there are two lines of singularity emerging from the north and south pole of $\mathbb{P}^{1}$, the angle between them being given by the mass of the adjoint field. A set of non-compact $\mathbb{P}^{1}$ cycles with D5 branes wrapping them end on these lines. Depending on their orientation, their end point on the north pole line describes the mass of the corresponding field while the end point on the south pole line describes a vev, or vice versa.

As any geometry containing a conifold singularity, this one exhibits a geometric transition similar to the standard one. Because of the presence of the D-branes on the non-compact cycles there is more information which needs to be taken through the transition.

\section{Geometric transition with fundamental fields}

The issue of introducing fundamental matter in the geometric transition has been considered in [3, 11, 31]; however, the analysis applies only to a theory with all quarks integrated out (i.e. they are all massive) and only for an infinitely massive $\mathcal{N}=2$ adjoint field. The goal of the present section is to relax these assumptions and recover the much richer set of results described in section 1.1. In particular, since the low energy theory is described in terms of mesons, an essential ingredient is their geometric interpretation.

There are two different ways of describing this. One way is to start from the brane configuration described above, lift it to $M$-theory and then map the results to the deformed geometry, paying particular attention to the vev-s of the fundamental fields. An alternative way is to start from a brane configuration describing a field theory with bifundamental fields which reduces to the theory of interest in a certain limit, describe the transition for its associated geometry and then take the appropriate limit at the end. The issue of vev-s for the bifundamental fields was discussed for the brane configurations in [51] and in the context of the geometric transition in [12]. The former line of reasoning emphasizes the behavior of the flavor branes under the geometric transition while somewhat obscuring the precise identification of the vevs of the fundamental fields. The latter argument identifies more clearly the vev of the fundamental fields in the deformed geometry while somewhat obscuring the behavior of flavor branes under the transition. For these reasons as well as for others which will become clear in section [5. we will describe both approaches.

Starting with the brane configuration in figure 1, the strong coupling limit 
is understood as lifting it to $M$-theory together with taking the separation between the two NS5 branes to zero. Arguments similar to those in [10-13] imply that the brane configuration becomes an M5 brane with the world volume [45, 46, 49] given by the curve

$$
y \hat{x}=\zeta \quad \hat{x}=x-\frac{y}{4 m_{a d j}}
$$

where $\hat{x}$ and $y$ are the coordinates along the two NS5 branes while $x$ is the coordinate along the dashed line in figure 1. As $x \rightarrow \infty$ we have either $y=0$ or $y \rightarrow m_{a d j} x$. In these coordinates $y$ is the coordinate along the dashed line, $y=x_{8}+i x_{9}$, while $x$ is the coordinate along $N S 5, x=x_{4}+i x_{5}$. The coefficient of $y^{2}$ can be freely adjusted to any non vanishing value, while remaining proportional to the mass of the adjoint field. We will use this freedom to identify $m_{a d j}$ with the mass parameter of the adjoint field.

The $\mathrm{M}$ theory lift of a D4 brane describing a field in the fundamental representation is a cigar-shaped $M 5$ brane which intersects the curve (25) in exactly one point, say $P$. As this point is constrained to lie on (25) only one of its coordinates can be arbitrarily chosen. This is consistent with the field theory expectation that, given the superpotential and a mass parameter there is a discrete set of choices for the expectation value of the meson field. Conversely, given the superpotential and an expectation value of the meson field, the mass parameter is uniquely determined. In type IIA language, this represents a set of semi-infinite D4 branes ending on an NS5 brane whose world volume is given by (25).

The discussion in the previous section suggests that the coordinate of $P$ along the $x$ direction equals the mass of the corresponding field while its coordinate along the $y$ direction represents the expectation value of the meson field built out of the corresponding field. Therefore, the strong coupling/Mtheory analog of the "integrating in/out" procedure of 42] represents the transition between the two choices of which one of the two coordinates of the point $P$ is fixed as "boundary condition".

We want to emphasize that during the transition only the compact $\mathbb{P}^{1}$ cycle shrinks, but the non-compact 2-cycles remain unchanged. In type IIA theory this represents the fact that at strong coupling there still exist semiinfinite D4 branes which end on the NS5 whose world volume is the curve (25).

Let us now turn to the other description of the geometric transition with flavor fields. The starting point is a theory with $U\left(N_{c}\right) \times U\left(N_{f}^{m}\right) \times U\left(N_{f}^{M}\right)$ 
as gauge group. An $\mathcal{N}=1$ brane configuration realizing this theory involves three NS5 branes, say $\mathrm{A}, \mathrm{B}$ and $\mathrm{C}$, at different points in the $x^{6}$ direction, whose projection on a $(x, y)$ plane forms a triangle, with corners denoted by $I_{A B}, I_{A C}$ and $I_{B C}$, respectively. Along the $x^{6}$ direction and at each corner of this triangle lie $N_{c}, N_{f}^{m}$ and $N_{f}^{M} \mathrm{D} 4$ branes, respectively. For definiteness, let us assume that there are $N_{c}$ between B and C, $N_{f}^{m}$ between A and B and $N_{f}^{M}$ between $\mathrm{A}$ and $\mathrm{C}$. This brane configuration was analyzed in detail in [56] where it was obtained by rotating an $\mathcal{N}=2$ brane configuration describing a gauge theory with gauge group $U\left(N_{c}+N_{f}^{M}\right) \times U\left(N_{f}^{m}+N_{f}^{M}\right)$ and bifundamental fields. Among other things, it was shown that the distance measured along the $\mathrm{B}$ brane between $I_{A B}$ and $I_{B C}$ is equated with the mass of the bifundamental fields while the distance measured in the direction orthogonal to the $\mathrm{B}$ brane between $I_{B C}$ and $I_{A C}$ is equated with the vev of the off-diagonal components of the scalar field in the adjoint representation which break $U\left(N_{c}+N_{f}^{M}\right)$ to $U\left(N_{c}\right)$.

To recover the brane configuration described in the previous section we take the A brane to infinity in the $x^{6}$ direction, without crossing the other NS branes (in what follows, we denote this process as a decoupling limit) ; in this limit the $U\left(N_{f}^{m}\right)$ and $U\left(N_{f}^{M}\right)$ gauge bosons become nondynamical, the gauge symmetry becomes global. Thus, the bifundamental fields survive as fundamentals of $U\left(N_{c}\right)$.

This setup was described geometrically in [12 in terms of a resolved $A_{2}$ singularity fibered over a plane. Among other things, it was shown that in the slices of fixed $x^{6}$ and $x^{7}$ there exists a 1-cycle and the inverse image under the projection onto these slices of the compact domain bounded by it is homotopic to an $S^{3}$. It was also shown that this cycle exists on both sides of the geometric transition. Since its size is proportional to the expectation value of the bifundamental fields, it can be used to give an invariant meaning for this expectation value in the deformed geometry ${ }^{4}$.

The geometric version of the fact that the brane $\mathrm{A}$ is taken to infinity is that the leftmost singularity line is taken to infinity without crossing the other two lines. In this limit two of the three $\mathbb{P}^{1}$ cycles decompactify and we recover the geometry described in the previous section. It is also clear

\footnotetext{
${ }^{4}$ The results of 12 describe the existence of two types of deformations in the deformed geometry. The first type are the "normalizable deformations" and correspond to dynamical quantities in field theory (e.g. the glueball superfield). The second type are the "nonnormalizable deformations" and correspond to non-dynamical quantities in field theory (as the vev of the bifundamental fields).
} 
that this limit can be taken as long as no geometric transition occurs for the 2-cycles which decompactify. Indeed, the geometric transition is the geometric version of the strong coupling limit while the decompactification is the geometric image of a small coupling limit.

In the resolved geometry, the decoupling limit leads to a degeneration of the "non-normalizable" $S^{3}$ cycle into an infinitely thin and infinitely long submanifold which touches all $\mathbb{P}^{1}$ cycles. Its projection onto the left-most line of singularities describes the mass of the massive flavor fields while its projection onto the direction orthogonal to it is proportional to the meson expectation value.

If the decoupling limit is taken after a geometric transition occurs for the $\mathbb{P}^{1}$ cycle between the two rightmost singularity lines, the "non-normalizable" $S^{3}$ cycle degenerates into an infinitely thin and infinitely long submanifold which touches the special Lagrangian cycle and the noncompact $\mathbb{P}^{1}$ cycles.

In terms of brane configurations, the two pictures correspond to moving the brane A to infinity before or after the NS5 branes B and C are deformed into a unique one. From this point of view it it clear that the decoupling limit and the deformation of the $N S 5$ branes commute. In geometric terms the two pictures correspond to decompactifying two $\mathbb{P}^{1}$ cycles before and after a geometric transition occurs for the third one; here, the decompactification

commutes with the geometric transition because of the geometric nature of each process.

Comparing the two pictures implies that the mass of the massive fields is given by the distance between the special Lagrangian cycle and the noncompact $\mathbb{P}^{1}$ cycle ending above the point $I_{A B}$. Denoting this direction by $x$, the vev of the remaining fields transforming in the fundamental representation of the gauge group is given by the projection of the distance between the special Lagrangian cycle and the noncompact $\mathbb{P}^{1}$ cycle ending above the point $I_{A C}$ onto the normal to $x$. This sharpens the identifications suggested by the first description of flavor fields.

\section{Effective superpotential at strong coupling}

After having discussed all the details of the geometric transition for an adjoint field of finite mass as well as for massive and massless flavor fields let us proceed to the computation of the effective superpotential. In this section we recover the gauge theory results (4) (or rather their form when the glueball 
superfield is included) from the deformed geometry with branes and fluxes. In the next section we will find the same results by expressing the computations in terms of a matrix model.

Thus, the starting point is a closed string background given by the deformed conifold in the coordinates in which its defining equation is:

$$
p q+y\left(x-\frac{y}{4 m_{A}}\right)=\zeta .
$$

In this geometry there exist $\mathrm{D} 5_{m}$ branes wrapping the noncompact cycles defined by the equation $q=0$ and boundary condition ${ }^{5} x(p \rightarrow \infty)=x_{*}$ as well as D $5_{M}$ defined by the same equation $q=0$ but a different boundary condition $y(p \rightarrow \infty)=y_{*}$.

As discussed in [3], the superpotential of the gauge theory dual to a configuration of fluxes and branes consists of two parts. The first part represents the contribution of fluxes and it is given by the GVW superpotential:

$$
W_{F}=\int \Omega \wedge F
$$

The second part consists of the contribution of branes. The theory living on the part of the branes wrapping the cycles is given by the holomorphic Chern-Simons action [52]. Their contribution to the superpotential can be computed by evaluating this action on a (generic) classical field configuration. This operation, which essentially integrates out at the classical level the fluctuations around the classical solution, describes the obstructions to the deformation of the branes. As we are interested in evaluating this action on a non-compact brane, the boundary conditions at infinity are kept fixed.

In the context of the conifold geometry describing an infinitely massive adjoint field and for branes describing massive flavor fields, both these contributions were computed in 3. We will extend this computation to describe a finite mass parameter for the adjoint field as well as flavor fields which develop large expectation values for their corresponding mesons.

To evaluate the superpotential (27) one usually writes it in terms of periods of $\Omega$ as well as fluxes through the dual cycles.

$$
W_{F}=\int_{A} \Omega \int_{B} H_{N S}-\int_{B} \Omega \int_{A} F_{R R}=\tau S-N_{c} \Pi
$$

\footnotetext{
${ }^{5}$ As described in [4], it is necessary to impose a boundary condition only in one of the $x$ or $y$ direction, as the other one is determined by equation (26).
} 
where

$$
S=\int_{A} \Omega \quad \Pi=\int_{B} \Omega \quad \tau=\int_{B} H_{N S} \quad N_{c}=\int_{A} F_{R R}
$$

The periods of $\Omega$ over compact cycles are invariant under changes of coordinates which do not change the complex structure. Thus, it is easy to see that the relation between $S$ and the deformation parameter $\zeta$ is identical to the one in the case of infinite mass for the adjoint field. Indeed, introducing the coordinates

$$
u=\sqrt{m_{A}} x \quad v=\frac{y}{2 \sqrt{m_{A}}}-\sqrt{m_{A}} x
$$

one can write the equation (26) as the usual big resolution of the conifold:

$$
u^{2}-v^{2}=\zeta
$$

This change of coordinates is holomorphic. Therefore, by writing the cycle as a 2 -sphere fibered over a segment, we find [3]:

$$
S=\int_{A} \Omega=\int_{-\zeta^{1 / 2}}^{\zeta^{1 / 2}} \sqrt{u^{2}-\zeta} d u=\frac{\zeta}{4}
$$

This is however not the case for periods over non-compact cycles. Indeed, the corresponding integrals are defined with a cutoff which changes under coordinate transformations. In the $(u, v)$ coordinates, the $B$-cycle can be defined as an $S^{2}$ fibration over a curve starting at $u=\sqrt{\zeta}$ and ending at some cutoff. However, we are interested in the periods computed in the $(x, y)$ coordinates and thus the cutoff in the $u$-plane should be derived from a more fundamental cutoff in the $x$-plane. The two cutoffs are related by (30); thus, the period integral defining $\Pi$ for finite mass for the adjoint field is:

$$
\Pi=\int_{\zeta^{1 / 2}}^{\Lambda_{0} \sqrt{m_{A}}} \sqrt{u^{2}-\zeta} d u=\frac{1}{2} \Lambda_{0}^{2} m_{A}+\left[-\frac{1}{4} \zeta-\frac{1}{4} \zeta \ln \frac{\Lambda_{0}^{2} m_{A}}{S}\right]+\mathcal{O}\left(\frac{1}{\Lambda_{0}}\right)
$$

Ignoring the terms which are polynomially divergent as the cutoff is taken to infinity, it follows that the GVW superpotential is

$$
W_{F}=S\left(\ln \frac{\Lambda^{2 N_{c}} m_{A}^{N_{c}}}{S^{N_{c}}}+N_{c}\right)
$$


where we also used the usual definition for the dynamical scale in terms of the cutoff and the gauge coupling (a.k.a. "dimensional transmutation")

$$
\Lambda^{2 N_{c}}=e^{-\tau} \Lambda_{0}^{2 N_{c}}
$$

Equation (34) is indeed the correct gauge-theoretic expressions for energy scales less than $m_{A}$ : the adjoint field is integrated out and its mass contributes to the dynamical scale:

$$
\Lambda_{\mathcal{N}=1}^{3 N_{c}}=\Lambda_{\mathcal{N}=2}^{2 N_{c}} m_{A}^{N_{c}}
$$

We now turn to the contribution of the D5 branes describing the fields charged under global symmetry groups. As described above, they contribute to the effective superpotential an amount equal to the holomorphic ChernSimons action (which is the theory living on the part of the brane wrapping the cycle) evaluated on a representative of the homology class of the noncompact 2-cycles with generic moduli dependence.

As in the case of the $B$-cycle described above, a proper definition for these cycles requires a choice of boundary conditions ${ }^{6}$.

To begin with, we recall that the Calabi-Yau space of interest is given by

$$
p q=F(x, y)
$$

embedded in $\mathbb{C}^{4}$. In this space, the noncompact cycles we are interested in are defined by [43]:

$$
\mathcal{C}: \quad F(x, y)=0 \quad q=0 \quad x(p \rightarrow \infty)=x_{*} \quad y(p \rightarrow \infty)=y_{*}
$$

where $x_{*}$ and $y_{*}$ represent boundary conditions and the function $F(x, y)$ is given by the right-hand-side of the equation (25). The coordinate parameterizing the cycle is denoted by $p$ while the position of the cycle in the total space is described by a point $\left(x_{*}, y_{*}\right)$ on the curve $\Sigma: F(x, y)=0$.

In 43 it was shown that the holomorphic Chern-Simons action can be written as:

$$
S=\int_{\mathcal{C}} \frac{d p}{p} \alpha d \beta=-\int_{\mathcal{C}} \frac{d p}{p} \beta d \alpha
$$

where $\alpha$ and $\beta$ are coordinates parameterizing the curve $\Sigma$ and describe one of the directions the cycle is allowed to fluctuate in. This in turn implies

\footnotetext{
${ }^{6}$ More formally, they are cycles in a nonstandard relative homology group, for which the constraint is given by the boundary conditions.
} 
that only one of them can be chosen independently as boundary condition; the other one is determined by the requirement that $(\alpha, \beta)$ lies on $\Sigma$. As the integral over $p$ factorizes, we are left with

$$
S=\int_{\Sigma} \alpha d \beta=-\int_{\Sigma} \beta d \alpha
$$

In choosing the boundary conditions at infinity we have to make sure that they represent a stable point on the curve at infinity. In other words, the intersection point between the cycle and $\Sigma_{*}=\Sigma(p \rightarrow \infty)$ should be one of the critical points on the direction along which boundary conditions are chosen.

With these clarifications, let us now evaluate (40) for $\mathrm{D} 5_{m}$. In $(x, y)$ coordinates, their position on the $x$ axis near the origin of the coordinate along the cycle describes the mass of the corresponding quarks. Thus, it is natural to fix the boundary conditions at infinity in these coordinates. We will nevertheless evaluate the action in the $(u, v)$ space.

As explained above, we fix the $x_{*}$ such that it is a critical point of $y(x)$. Fixing the origin on $\Sigma_{*}$ at $x=m$ and solving $F(x, y)=0$ for $y$ we find that one of the critical points is at infinity, which we regularize by introducing a cutoff $\Lambda_{0}$. Translating to the initial origin on $\Sigma$ we find that we must integrate over

$$
x \in\left[m, \Lambda_{0}+m\right] .
$$

This interval can easily be translated into an integration domain for $u$. Ignoring terms which are polynomially divergent as the regulator is removed as well as terms which vanish in this limit, it follows that the superpotential is:

$$
\begin{aligned}
& W_{m}=\frac{1}{2} \int_{m \sqrt{m_{A}}}^{\left(\Lambda_{0}+m\right) \sqrt{m_{A}}} \sqrt{u^{2}-\zeta} d u \\
& =-S\left[\frac{1}{2}+\frac{1}{4 k_{m} S}\left(\sqrt{1-4 k_{m} S}-1\right)-\ln \left(\frac{1}{2}+\frac{1}{2} \sqrt{1-4 k_{m} S}\right)\right]+S \ln \frac{m}{\Lambda_{0}}
\end{aligned}
$$

where we have introduced the notation

$$
k_{m}=\frac{1}{m^{2} m_{A}} .
$$

We can easily recover the results of [43] by taking the mass of the adjoint field to infinity, or rather equal to the cutoff. It is not hard to see that the 
only surviving term from the equation above is

$$
W_{m}\left(m_{A} \rightarrow \infty\right)=S \ln \frac{m}{\Lambda_{0}}
$$

Let us now consider the contribution of $\mathrm{D} 5_{M}$ to the effective superpotential. As in the previous situations, we will fix the boundary conditions in the $(x, y)$ space and then translate them to $(u, v)$. In the previous section we argued that the projection on the $y$ axis of the displacement of the $\mathrm{D} 4_{M}$ brane along $N S 5^{\prime}$ can be identified with the meson expectation value. Thus, we will consider a noncompact 2-cycle which ends at coordinate $y=4 \sqrt{2} i M{ }^{7}$

Determining the boundary condition at infinity is slightly more involved. First we solve the equation (25) for $x(y)$ :

$$
x=\frac{y^{2}+4 \zeta^{2} m_{A}^{2}}{4 m_{A} y} .
$$

From here we see that there are several critical points. To make connection with the brane picture, we would like to pick boundary conditions such that, as the coupling constant is decreased, the mesons will have a large expectation value. As there is no critical point at infinity for real values of $y$, we will choose the brane to end at the critical point at imaginary infinity in the $y$ direction.

$$
y_{*}=4 \sqrt{2} i\left(\Lambda_{0}^{2}\right) \quad x\left(y_{*}\right)=4 i \frac{\Lambda_{0}^{2}}{4 m_{A}}+\mathcal{O}\left(\frac{1}{\Lambda_{0}}\right) .
$$

Therefore, we have the following integration domain:

$$
y \in 4 \sqrt{2}\left[i \Lambda_{0}^{2}, i M\right]
$$

which in $(u, v)$ coordinates becomes

$$
v \in \frac{\sqrt{2}}{\sqrt{m_{A}}}\left[i \Lambda_{0}^{2}, i M\right]
$$

upon assuming that $M$ is large.

\footnotetext{
${ }^{7}$ The numerical factor can be traced to a similar factor in equation (25). It is related to a different choice of $y$ coordinate compared to 46].
} 
Thus, we are required to compute:

$$
\begin{aligned}
W_{M} & =\frac{1}{2} \int_{i \frac{\sqrt{2} \Lambda_{0}^{2}}{\sqrt{m_{A}}}}^{i \frac{\sqrt{2} M}{\sqrt{m_{A}}}} \sqrt{v^{2}+\zeta} d v=-\frac{1}{2} \int_{\frac{\sqrt{2} \Lambda_{0}^{2}}{\sqrt{m_{A}}}}^{\frac{\sqrt{2} M}{\sqrt{m A}}} \sqrt{v^{2}-\zeta} d v \\
& =-\frac{M^{2}}{2 m_{A}}+S \ln \frac{\Lambda_{0}^{2}}{M}
\end{aligned}
$$

where we have ignored terms of order $\frac{1}{\Lambda}$ and $\frac{1}{M}$.

We are now in position to construct the full superpotential. However, in combining equations (34), (42) and (49) we have to be careful in counting the $R R$ flux through the $A$-cycle. As we saw in an earlier section, for small coupling, an expectation value for the meson field is equated with an expectation value for the fundamental fields. If the expectation value of the meson is larger than the dynamical scale but smaller that the cutoff, a similar identification is possible ${ }^{8}$. Since we assumed $M$ to be large and comparable to $\Lambda_{0}^{2}$, this is the regime we are studying. Thus, the brane picture applies without modification and the rank of the gauge group is smaller compared to the pure gauge theory by an amount equal to the rank of the expectation value of the meson matrix. The full superpotential is therefore:

$$
\begin{aligned}
W_{\text {full }} & =S\left(\ln \frac{\Lambda_{0}^{2\left(N_{c}-N_{f}^{M}\right)} m_{A}^{N_{c}-N_{f}^{M}}}{S^{N_{c}-N_{f}^{M}}}+N_{c}-N_{f}^{M}\right)-\tau S \\
& +S \ln \frac{\Lambda_{0}^{2 N_{f}^{M}}}{\operatorname{det} M}-\frac{1}{2 m_{A}} \operatorname{Tr}\left[M^{2}\right]+S \ln \frac{\operatorname{det} m}{\Lambda_{0}^{N_{f}^{m}}} \\
& -\sum_{m} S\left[\frac{1}{2}+\frac{1}{4 k_{m} S}\left(\sqrt{1-4 k_{m} S}-1\right)-\ln \left(\frac{1}{2}+\frac{1}{2} \sqrt{1-4 k_{m} S}\right)\right] \\
& =-\frac{1}{2 m_{A}} \operatorname{Tr}\left[M^{2}\right]+S\left(\ln \frac{\Lambda^{3 N_{c}-N_{f}} \operatorname{det} m}{S^{N_{c}-N_{f}^{M}} \operatorname{det} M}+N_{c}-N_{f}^{M}\right) \\
& -\sum_{m} S\left[\frac{1}{2}+\frac{1}{4 k_{m} S}\left(\sqrt{1-4 k_{m} S}-1\right)-\ln \left(\frac{1}{2}+\frac{1}{2} \sqrt{1-4 k_{m} S}\right)\right]
\end{aligned}
$$

\footnotetext{
${ }^{8}$ Since the quantum contribution to the expectation value of the meson is equal to the dynamical scale up to coefficients of order one it follows that, if it is larger than $\Lambda$, it must be generated at the classical level
} 
where $k_{m}=m^{2} m_{A}, \Lambda$ is the dynamical scale at which the adjoint field is integrated out, $N_{f}^{M}$ is the number of fundamental fields combined into mesons, $N_{f}^{m}$ is the number of massive fundamental fields and $N_{f}=N_{f}^{M}+N_{f}^{m}$.

\section{Effective superpotential at weak coupling; Matrix Models}

In this section we recover the field theoretic effective superpotential in the resolved geometry and provide a geometric justification of certain proposals which appeared in the relation between the matrix models with massless flavors and gauge theory.

\subsection{Review of the results for pure gauge theories}

The large $N$ duality between open strings (branes) on the resolved geometry and closed strings (fluxes) on the deformed geometry was the starting point of the Dijkgraaf-Vafa conjecture. They argued that the effective superpotential of the gauge theory living on the non-compact part of D5 branes wrapping compact 2-cycles in the resolved geometry is given by the free energy of the matrix model built with the superpotential of the gauge theory and that this free energy is equal to the one of the topological IIB superstrings on the deformed side.

In the case of the small resolution of the conifold, the arguments for this bold conjecture rely on the fact that the fields living on the 2-cycles are governed by the holomorphic Chern-Simons theory [52] as well as on the fact that a field theory superpotential can be included in this theory by simply shifting the action by an amount equal to the product between the Kähler class and the superpotential evaluated on 0-form deformations [55].

$$
Z=\int d \Phi_{0} d \Phi_{1} e^{\int_{\mathcal{C}} \Phi_{0} \bar{D} \Phi_{1}+W\left(\Phi_{0}\right) \omega}
$$

Then, the equations of motion allows one to set $\Phi_{1}=0$, as well as restrict $\Phi_{0}$ to the zero mode. Thus, the partition function reduces to just an integral over matrices:

$$
Z=\int d \Phi e^{-\frac{1}{g_{s}} \operatorname{Tr} W(\Phi)}
$$

The assumptions of this proposal include the identification of the glueball superfield with the 't Hooft coupling of the matrix model: $S=N g_{s}$. It is 
important to emphasize that the dimension $N$ of the matrices appearing in the matrix model is unrelated to the rank of the gauge group $N_{c}$.

The original Dijkgraaf-Vafa conjecture was stated for complex deformations of an $\mathcal{N}=2, A_{1}$ singularity. Similar results hold in the case of other $\mathcal{N}=2$ singularities, e.g. those which lead to quiver gauge theories. In that case, besides the integral of the adjoint fields as in (52), some extra terms are required for integrating the bifundamental fields and the Chern-Simons action is simplified due to localization on the lowest lying modes. Thus, one adds to the superpotential:

$$
W_{b i f}=\sum_{i} \operatorname{Tr}\left[Q_{i, i+1} \Phi_{i+1} \tilde{Q}_{i+1, i}\right]+\operatorname{Tr}\left[\tilde{Q}_{i+1, i} \Phi_{i} Q_{i, i+1}\right]
$$

We now turn to the discussion of fields in the fundamental representation and discuss the geometric justification of the various procedures of dealing with massless quarks [24, 30, 39].

\subsection{Massive and massless matrix models}

The geometry and matrix models for gauge theories with massive fundamental matter were related in several papers [22, 35, 31] (see also [15, 33] for the case of bifundamental matter). In [31] it was suggested that all the D5 branes are replaced by $R R$ fluxes which would mean that all the 2-cycles shrink and are replaced by $S^{3}$ with flux.

As described in section 3, the simplest way to deal, in a similar way, with both fundamental and bifundamental fields with Yukawa-type superpotentials is to start with the product of two gauge groups $U\left(N_{c}\right) \times U\left(N_{f}\right)$ with bifundamental fields and to take the coupling constant of the $U\left(N_{f}\right)$ group to zero. Thus, this symmetry becomes a global one and the bifundamental fields transform in the fundamental or anti-fundamental representation of the remaining gauge group. This method was used in the DV context in 35] and we will adjust it to our case.

It is however worth pointing out that this limit can be interpreted geometrically as a "partial geometric transition". Indeed, the size of the $\mathbb{P}^{1}$ cycles wrapped by D5 branes is proportional to the inverse of the coupling constant of the corresponding factor of the gauge group. Thus, the geometric transition occurs only for the cycle wrapped by the branes generating the $U\left(N_{c}\right)$ gauge group, while the others remain as $\mathbb{P}^{1}$ cycles; the vanishing coupling constant limit corresponds to decompactifying them. 
Let us begin with the $\mathcal{N}=2$ theory with product gauge group $U\left(N_{c}\right) \times$ $U\left(N_{f}\right)$, which is geometrically engineered as a resolved $A_{2}$ singularity with $N_{c}$ D5 branes on one $\mathbb{P}^{1}$ and $N_{f}$ D5 branes on the other $\mathbb{P}^{1}$. We then break half of the supersymmetry to by adding a mass term for the adjoint fields. More generally, one can add an arbitrary potential for them, but this does not modify the discussion. As discussed in section 3, there is also an $S^{3}$ cycle whose size is proportional to the vevs of the massless fundamental fields.

The corresponding matrix model is [15]:

$$
Z=\int d \Phi_{1} d \Phi_{2} d Q d \tilde{Q} e^{-\operatorname{Tr} W\left(\Phi_{1}, \Phi_{2}, Q, \tilde{Q}\right)}
$$

where $\Phi_{i}$ are $M_{i} \times M_{i}$ matrices, $Q$ is $M_{1} \times M_{2}$ matrix and

$$
\begin{array}{r}
W\left(\Phi_{1}, \Phi_{2}, Q, \tilde{Q}\right)=\frac{1}{g_{1}} W_{1}\left(\Phi_{1}\right)+\frac{1}{g_{2}} W_{2}\left(\Phi_{2}\right)+ \\
\operatorname{Tr}\left[Q \Phi_{2} \tilde{Q}-\tilde{Q} \Phi_{1} Q\right]
\end{array}
$$

$W_{i}\left(\Phi_{i}\right)$ being polynomials of $\Phi_{i}$.

The above superpotential breaks supersymmetry to $\mathcal{N}=1$ and the moduli space is described by the expectations values for the adjoint fields $\Phi_{i}$ and for the bifundamental fields $Q$ and $\tilde{Q}$. Taking the limit of vanishing $g_{2}$ freezes $\Phi_{2}$ to one of the minima of $W_{2}$. Since we are interested in both massive and massless flavor fields, we assume that only part of the diagonal entries of $\Phi_{2}$ are nonvanishing. As the diagonal entries of $\Phi_{2}$ give the mass for the quarks, we have then a splitting of $Q$ and $\tilde{Q}$ into massive and massless fields, the potential for the matrix model above being

$$
V_{M M}(\Phi, Q, \tilde{Q})=W(\Phi)+\sum_{i=1}^{N_{f}}\left(Q_{i} \Phi \tilde{Q}_{i}\right)+\sum_{i=1}^{N_{j}^{m}} m_{i} Q_{i} \tilde{Q}_{i}
$$

There is, however, more information which can be obtained from the description of massless flavors in the previous sections. In particular, we had to impose boundary conditions on the cycles describing both massive and massless flavors. The mass term in the previous equation can be interpreted in that setup as arising because the noncompact 2-cycle describing massive fundamental fields ends at one of the nonvanishing minima of $W_{2}$.

It is however clear that the above superpotential does not take into account the boundary conditions required for the cycle describing massless 
flavor fields. In the previous section we imposed the condition that the noncompact 2-cycles describing the massless fields end on the curve $\Sigma$ which is orthogonal to them at the points fixed by the eigenvalues of the meson matrix. An equivalent way of stating this boundary condition is that the holomorphic Chern-Simons action is evaluated with the constraint that the meson eigenvalues are fixed.

We can now supplement the potential $V_{M M}$ with the appropriate constraint. In the weak coupling regime the meson is just a bilinear in quarks and antiquarks. Furthermore, one can perform an $S U\left(N_{f}\right)$ global rotation and replace the constraint that the eigenvalues of $Q_{i} \tilde{Q}_{j}$ are fixed with the requirement that $Q_{i} \tilde{Q}_{j}$ is some fixed matrix. In principle, its eigenvalues should be equal to those which appear in the original constraint. However, as they were arbitrary, the corresponding matrix is arbitrary. Thus, the partition function of matrix model with massive and massless flavors is:

$$
Z=\mathcal{N} \int d \Phi d Q^{m} d \tilde{Q}^{m} d Q^{M} d \tilde{Q}^{M} \delta\left(Q_{i}^{M} \tilde{Q}_{j}^{M}-M_{i j}\right) e^{-V_{M M}(\Phi, Q, \tilde{Q})}
$$

The normalization of this partition function requires division by the inverse volume of the matrix model "gauge group" $U(N)$. As in the original DV proposal, this can be interpreted as being part of the planar and boundaryless free energy. Thus, its contribution to the superpotential is its derivative multiplied by the rank of the gauge group (the unbroken as well as the broken part!).

The equation (58) recovers the suggestion [24] for the inclusion of massless quarks in the matrix model, and is also equivalent [39] with the suggestion of [30] that one first deforms the matrix model by mass terms and then takes the massless limit. This can be easily seen by using an integral representation for the $\delta$-function in equation (58) and noticing that the new variable plays the role of mass parameter for the quarks $Q^{M}$ and $\tilde{Q}^{M}$.

For illustration purposes, let us briefly analyze the case of a quadratic superpotential for the adjoint field and recover equation (50). We will also concentrate on the terms linear in $N_{f}$. In the cases covered by our analysis, this can be understood as arising from the large $N$ limit for the matrix model gauge group.

The easiest way to go about computing the free energy in this regime is to represent it as a sum of vacuum Feynman diagrams and furthermore notice that any diagram contains exactly one species of quarks. Thus, the free energy receives two independent contributions, one from the massive quarks 
and the other from the massless ones. The integral over massive quarks is computed as in 21] and gives

$$
Z=e^{-\frac{1}{g_{s}} \mathcal{F}_{\text {massive }}} \int d \Phi d Q^{M} d \tilde{Q}^{M} \delta\left(Q_{i}^{M} \tilde{Q}_{j}^{M}-M_{i j}\right) e^{\left.-\frac{1}{g_{s}} \operatorname{Tr}\left[Q^{M} \Phi \tilde{Q}^{M}+\frac{1}{2} m_{A} \Phi^{2}\right)\right]}
$$

where

$$
\begin{aligned}
\mathcal{F}_{\text {massive }} & =\sum_{i=1}^{N_{f}^{m}} \mathcal{F}\left(m_{i}\right) \\
\mathcal{F}(m) & =S\left[-\frac{1}{2}-\frac{1}{4 \alpha_{m} S}\left(\sqrt{1-4 \alpha_{m} S}-1\right)+\ln \left[\frac{1}{2}+\frac{1}{2} \sqrt{1-4 \alpha_{m} S}\right]\right]
\end{aligned}
$$

and $\alpha_{m}=\frac{1}{m_{A} m^{2}}$.

The next step is to integrate out the adjoint field, as a gaussian integral which implies the appearance of $\operatorname{Tr}\left[\left(Q^{M} \tilde{Q}^{M}\right)^{2}\right]$. The remaining integrand is then expressed only in terms of the bilinear $\left(Q^{M} \tilde{Q}^{M}\right)$ and it can be pulled out of the integral because of the $\delta$-function. Furthermore, this integral is also part of the planar and boundaryless free energy. As its contribution to the superpotential is slightly different than the one of flavor fields, we will leave it aside for the moment. Surely enough, we will add it back at the end. We are therefore left with:

$$
Z=e^{-\frac{1}{g_{s}}\left(\mathcal{F}_{\text {massive }}+\frac{1}{m_{A} m^{2}} \operatorname{Tr}\left[M^{2}\right]\right)} \int d Q^{M} d \tilde{Q}^{M} \delta\left(Q_{i}^{M} \tilde{Q}_{j}^{M}-M_{i j}\right)
$$

where $\Lambda$ is a cutoff introduced here for dimensional reasons.

The remaining integral was performed in (24]) and yields:

$$
\int d Q^{M} d \tilde{Q}^{M} \delta\left(Q_{i}^{M} \tilde{Q}_{j}^{M}-M_{i j}\right)=e^{\frac{1}{g_{s}}\left[S \ln \left(\operatorname{det} M / \Lambda^{2 N_{f}^{M}}\right)-N_{f}^{M} S \ln \frac{S}{\Lambda^{3}}\right]} .
$$

Combining all the pieces together, the result is that the part of the free energy of the matrix model arising from the integration over fields is given by:

$$
-g_{s} \ln Z=\sum_{i=1}^{N_{f}^{m}} \mathcal{F}\left(m_{i}\right)+S\left[\ln \frac{\Lambda^{2 N_{f}^{M}}}{\operatorname{det} M}-N_{f}^{M}\right]
$$

where $\Lambda$ is a cutoff. Clearly the $U\left(N_{f}\right)$ invariance can be restored by replacing the product of eigenvalues of the meson matrix with its determinant. 
Adding to this the contribution of the normalization coefficient $\mathcal{N}$ (i.e. the Veneziano-Yankielowicz superpotential for the group $\left.U\left(N_{c}\right)\right)$ as well as the contribution of the integral over the adjoint field recovers equation (44), proving that the matrix model (58) describes the full nonperturbative physics of the gauge theory.

\section{Conclusions}

The main goal of our work was to fill certain gaps in understanding the relation between the gauge theories, geometry and matrix models for field theories with fields transforming in the fundamental representation of the gauge group.

We described in detail the geometric construction of a supersymmetrybreaking mass term of finite size for the adjoint field in the simplest $\mathcal{N}=$ 2 theory and analyzed the inclusion of massive and massless fields in the fundamental representation. The gauge theory described by this construction is $\mathcal{N}=1$ SQCD with massive and massless quarks coupled with an adjoint field of finite mass through a Yukawa coupling. Analyzing the geometric transition for this construction we computed the effective superpotential for this theory emphasizing the contribution of massless quarks as well as that of the finite adjoint mass.

Using the information we gained from this analysis we reconsidered the geometry prior to the geometric transition. For theories without fields transforming in the fundamental representation this was the starting point which lead to the DV proposal. While the inclusion of massive quarks in this framework was easily achieved without reference to geometry, certain difficulties were encountered in dealing with massless ones. The two solutions to this problem, proposed in [24] and [30] on a field-theoretic basis only, were shown to be equivalent in 39. From our analysis we see that this identification appears naturally from the geometrical picture and its relation to brane configurations. As was emphasized before (see [10-13]), the brane configurations represent a very useful tool in the description of geometric transitions and even more so in the light of the new correspondences between geometry, field theories and matrix models.

Finally, we illustrated the use of the matrix model we constructed and recovered the effective superpotential computed from geometric and topological considerations and found an exact agreement. 
There are several directions which can be pursued further. As we describe the case of massless flavors, it would be interesting to use D6 branes instead of D4 branes. One immediate problem is pushing them through the T-duality which gives a geometric description to the brane configuration, as naively they become D5 branes passing through the interior of the $P^{1}$, which does not belong to the space.

Another interesting direction is to consider brane configurations corresponding to chiral theories; T-duality transformations would map them to geometries which cannot be obtained from $\mathcal{N}=2$ ones by deformations. If possible, these would become an extension of the original conjectures to the chiral case. For many models of brane configurations see [49].

\section{Acknowledgments}

R.T. would like to thank Keshav Dasgupta and Kyungho Oh for discussions and collaboration on related issues. R.R. and J.W. thank Oliver DeWolfe for discussions. R.R. is supported in part by DOE under Grant No. 91ER40618 and in part by the NSF under Grant No. PHY00-98395. R.T. is supported by the DOE grant No. DE-AC03-76SF00098, the NSF under Grant No. PHY0098840 and by the Berkeley Center for Theoretical Physics. J.W. is supported in part by the NSF under Grant No. PHY99-07949. Any opinions, findings, and conclusions or recommendations expressed in this material are those of the authors and do not necessarily reflect the views of the National Science Foundation.

\section{References}

[1] R. Gopakumar and C. Vafa, Adv. Theor. Math. Phys. 3, 1415 (1999), hep-th/9811131.

[2] C. Vafa, J. Math. Phys. 42, 2798 (2001), hep-th/0008142.

[3] F. Cachazo, K. A. Intriligator and C. Vafa, Nucl. Phys. B 603, 3 (2001), hep-th/0103067

[4] J. D. Edelstein, K. Oh and R. Tatar, JHEP 0105, 009 (2001), hep-th/0104037. 
[5] F. Cachazo, S. Katz and C. Vafa, hep-th/0108120.

[6] F. Cachazo, B. Fiol, K. A. Intriligator, S. Katz and C. Vafa, Nucl. Phys. B 628, 3 (2002), hep-th/0110028

[7] B. Fiol, JHEP 0207, 058 (2002), hep-th/0205155.

[8] H. Fuji and Y. Ookouchi, hep-th/0205301.

[9] F. Cachazo and C. Vafa, hep-th/0206017.

[10] K. Dasgupta, K. Oh and R. Tatar, Nucl. Phys. B 610, 331 (2001), hep-th/0105066.

[11] K. Dasgupta, K. Oh and R. Tatar, JHEP 0208, 026 (2002), hep-th/0106040.

[12] K. h. Oh and R. Tatar, hep-th/0112040, Adv. Theor. Math. Phys. 6 (2002) 141-196

[13] K. Dasgupta, K. h. Oh, J. Park and R. Tatar, JHEP 0201, 031 (2002), hep-th/0110050.

[14] R. Dijkgraaf and C. Vafa, Nucl. Phys. B 644, 3 (2002), hep-th/0206255.

[15] R. Dijkgraaf and C. Vafa, Nucl. Phys. B 644, 21 (2002), hep-th/0207106.

[16] R. Dijkgraaf and C. Vafa, hep-th/0208048.

[17] R. Dijkgraaf, M. T. Grisaru, C. S. Lam, C. Vafa and D. Zanon, hep-th/0211017.

[18] F. Cachazo, M. R. Douglas, N. Seiberg and E. Witten, arXiv:hep-th/0211170.

[19] F. Cachazo, N. Seiberg and E. Witten, arXiv:hep-th/0301006.

[20] D. Berenstein, Phys. Lett. B 552, 255 (2003), hep-th/0210183.

[21] R. Argurio, V. L. Campos, G. Ferretti and R. Heise, hep-th/0210291.

[22] J. McGreevy, hep-th/0211009.

[23] H. Suzuki, hep-th/0211052, hep-th/0212121. 
[24] Y. Demasure and R. A. Janik, hep-th/0211082.

[25] I. Bena and R. Roiban, hep-th/0211075, to appear in Phys.Let.B.

[26] Y. Tachikawa, hep-th/0211189, hep-th/0211274.

[27] B. Feng, hep-th/0211202 B. Feng and Y. H. He, hep-th/0211234

[28] R. Argurio, V. L. Campos, G. Ferretti and R. Heise, Phys. Lett. B 553, 332 (2003), hep-th/0211249.

[29] S. G. Naculich, H. J. Schnitzer and N. Wyllard, JHEP 0301, 015 (2003), hep-th/0211254, hep-th/0211123.

[30] I. Bena, R. Roiban and R. Tatar, hep-th/0211271.

[31] Y. Ookouchi, hep-th/0211287.

[32] K. Ohta, hep-th/0212025.

[33] S. Seki, hep-th/0212079.

[34] I. Bena, S. de Haro and R. Roiban, hep-th/0212083.

[35] C. Hofman, hep-th/0212095.

[36] Y. Demasure and R. A. Janik, hep-th/0212212.

[37] N. Seiberg, hep-th/0212225.

[38] C. Ahn and S. Nam, hep-th/0212231; C. Ahn, hep-th/0301011.

[39] B. Feng, arXiv:hep-th/0212274.

[40] F. Ferrari, Nucl. Phys. B 648, 161 (2003), hep-th/0210135,

hep-th/0211069

H. Fuji and Y. Ookouchi, JHEP 0212, 067 (2002), hep-th/0210148:

R. Dijkgraaf, S. Gukov, V. A. Kazakov and C. Vafa, hep-th/0210238.

A. Gorsky, hep-th/0210281.

R. Gopakumar, hep-th/0211100,

R. Dijkgraaf, A. Neitzke and C. Vafa, hep-th/0211194. 
A. Klemm, M. Marino and S. Theisen, hep-th/0211216.

R. Dijkgraaf, A. Sinkovics and M. Temurhan, hep-th/0211241.

H. Itoyama and A. Morozov, hep-th/0211245.

S. K. Ashok, R. Corrado, N. Halmagyi, K. D. Kennaway and C. Romelsberger, hep-th/0211291.

B. Feng, hep-th/0212010.

H. Itoyama and A. Morozov, hep-th/0212032, hep-th/0301136.

R. A. Janik and N. A. Obers, Phys. Lett. B 553, 309 (2003) hep-th/0212069.

V. Balasubramanian, J. de Boer, B. Feng, Y. H. He, M. x. Huang, V. Jejjala and A. Naqvi, hep-th/0212082.

M. Matone, hep-th/0212253.

A. Dymarsky and V. Pestun, hep-th/0301135.

F. Ferrari, hep-th/0301157.

[41] N. Seiberg, Phys. Rev. D 49, 6857 (1994), hep-th/9402044.

[42] K. A. Intriligator, Phys. Lett. B 336, 409 (1994), hep-th/9407106.

[43] M. Aganagic and C. Vafa, arXiv:hep-th/0012041

[44] A. Hanany and E. Witten, Nucl. Phys. B 492, 152 (1997), hep-th/9611230.

[45] E. Witten, Nucl. Phys. B 500, 3 (1997), hep-th/9703166.

[46] K. Hori, H. Ooguri and Y. Oz, Adv. Theor. Math. Phys. 1, 1 (1998), hep-th/9706082.

[47] J. L. Barbon, Phys. Lett. B 402, 59 (1997); hep-th/9703051.

[48] A. Brandhuber, N. Itzhaki, V. Kaplunovsky, J. Sonnenschein and S. Yankielowicz, Phys. Lett. B 410, 27 (1997), hep-th/9706127.

[49] A. Giveon and D. Kutasov, Rev. Mod. Phys. 71, 983 (1999), hep-th/9802067.

[50] H. Ooguri and C. Vafa, Nucl. Phys. B 463, 55 (1996), hep-th/9511164. 
[51] J. H. Brodie and A. Hanany, Nucl. Phys. B 506, 157 (1997), hep-th/9704043.

[52] E. Witten, Prog. Math. 133, 637 (1995), hep-th/9207094.

[53] O. DeWolfe, D.Z. Freedman and H. Ooguri, Phys.Rev.D66:025009,2002; hep-th/0111135

[54] S. Gubser, N. Nekrasov and S. Shatashvili, JHEP 9905, 003 (1999), hep-th/9811230.

[55] S. Kachru, S. Katz, A. Lawrence and J. McGreevy

Phys. Rev. D 62, 026001 (2000), hep-th/9912151.

[56] A. Giveon and O. Pelc Nucl. Phys. B 512, 103 (1998), hep-th/9708168. 